\title{
Obesity in Older Adults and Associations with Cardiovascular Structure and Function
}

\author{
Yen How Tan ${ }^{a}$ Jun Pei Lim ${ }^{b}$ Wee Shiong Limb Fei Gao ${ }^{a, c}$ Louis L.Y. Teo ${ }^{a}$ c \\ See Hooi Ewe ${ }^{a, c}$ Bryan M.H. Keng ${ }^{a}$ Ru San Tan ${ }^{a, c}$ Woon-Puay Koh ${ }^{d}$ \\ Angela S. Koha, c \\ ${ }^{a}$ National Heart Centre Singapore, Singapore, Singapore; ${ }^{\text {t} T a n ~ T o c k ~ S e n g ~ H o s p i t a l, ~ S i n g a p o r e, ~ S i n g a p o r e ; ~}$ \\ 'Duke-NUS Medical School, Singapore, Singapore; ${ }^{\mathrm{d} H e a l t h y}$ Longevity Translational Research Programme, Yong Loo \\ Lin School of Medicine, National University of Singapore, Singapore, Singapore
}

\section{Keywords \\ Obesity · Older adults · Cardiovascular disesase · Ageing}

\begin{abstract}
Introduction: Body mass index (BMI), despite being widely used as a marker of obesity, fails to fully capture cardiovascular risks as it is an insufficient biomarker of abdominal adiposity, unlike waist circumference (WC). We aimed to characterize associations between BMI and WC with cardiovascular structure and function in older adults. Methods: Among an observational cohort study of a community of older adults, transthoracic echocardiography determined cardiovascular structure and function, while aerobic capacity was determined by peak oxygen uptake $\left(\mathrm{VO}_{2}\right)$ metrics. The cutoffs for obesity were $27.5 \mathrm{~kg} / \mathrm{m}^{2}$ for $\mathrm{BMI}$, and $>90 \mathrm{~cm}$ for males and $>80 \mathrm{~cm}$ for females for WC. Results: Of 970 older adults without cardiovascular disease (mean age $73 \pm 4$ years, 432 [44\%] males), 124 (12.8\%) were obese by BMI definition while 347 (35.7\%) were obese by WC definition. Interdefinitional agreement was fair (Cohen's $\mathrm{K}=0.345$ ). Unlike the BMI definition, participants defined as obese by WC were more likely to be women ( $65 \%$ vs. $50 \%, p<0.001)$, older (65 \pm 11 vs. $63 \pm 14$ years, $p=0.007)$, and had lower handgrip
\end{abstract}

strength ( $24 \pm 0.6$ vs. $26 \pm 0.4 \mathrm{~kg}, p=0.022$ ). Across BMI categories, high WC was associated with more impaired myocardial relaxation (E/A), and $\mathrm{VO}_{2}$ measurements (all $p<0.05$ ). Among those with low BMI, high WC was associated with larger left atrial (LA) volumes $(p=0.003)$. WC, but not $\mathrm{BMI}$, was independently associated with $E / A(\beta=-0.114$, SE $-0.114 \pm 0.024, p<0.001)$ in regression analysis. Conclusion: WC identified a higher prevalence of obesity, possibly related to central adiposity. Across BMI categories, WC identified more adverse measurements in E/A, aerobic capacity, and LA structure. Trial Registration: ClinicalTrials.gov Identifier: NCT02791139.

\section{Introduction}

Obesity and ageing are major health challenges of the 21 st century. Obesity increases the risk of death from any cause and from cardiovascular disease in adults, while age is a well-established cardiovascular risk factor. Although the body of evidence indicates that obese older subjects are prone to cardiovascular morbidity [1, 2], younger adults have higher relative risks associated with obesity

(C) 2022 The Author(s).

Published by S. Karger AG, Basel

This is an Open Access article licensed under the Creative Commons Attribution-NonCommercial-4.0 International License (CC BY-NC) (http://www.karger.com/Services/OpenAccessLicense), applicable to the online version of the article only. Usage and distribution for commercial purposes requires written permission.
Correspondence to:

Angela S. Koh, angela.koh.s.m@ singhealth.com.sg 
than older adults, based on body weight definitions of obesity [3]. This may lead to reduced emphasis on obesity as a risk factor among older adults [4]. However, body weight among older adults reflects a combination of overall health status and processes of aging-induced weight loss, such as sarcopenia [5]. This may explain lower relative risks associated with body mass index (BMI) definition of obesity among older adults, compared to younger adults. Therefore, the assessment of obesity based on BMI in older adults may inadequately identify older adults at risk of obesity-related cardiovascular disease.

Given the cardiometabolic effects of obesity on cardiovascular risks, waist circumference (WC) on the other hand, may enhance assessments of obesity among older adults. As a marker of central adiposity, measurement of WC is not influenced by limb sarcopenia, which is relevant among older adults with age-related sarcopenia. In addition, older adults with obesity have been recognized as a distinct metabolic phenotype (compared to older adults without obesity) that is associated with higher risks of cardiovascular disease [6]. Hypothetically, WC may have added value in identifying older adults with more adverse phenotypic alterations in cardiovascular structure and function, compared to BMI. Accordingly, we aimed to compare the relative prevalence and factors associated with obesity defined by WC vis-à-vis BMI, and to characterize their associations with cardiovascular structure and function in older adults without cardiovascular disease.

\section{Methods}

\section{Study Population}

The subjects were recruited from the Cardiac Ageing Study (CAS) [7], a prospective study initiated in 2014 that examines characteristics and determinants of cardiovascular function in elderly adults. CAS participants were recruited from the prospective, population-based cohort, the Singapore Chinese Health Study [8] and directly from the local community. The current study sample consisted of men and women who participated in the baseline CAS 2014-2017 examination who had no self-reported history of physician-diagnosed cardiovascular disease (such as coronary heart disease, atrial fibrillation), stroke, or cancer. Written informed consent was obtained from participants upon enrolment. The SingHealth Centralised Institutional Review Board (CIRC/2014/628/C) had approved the study protocol.

\section{Data Acquisition}

All participants were examined and interviewed on one study visit by trained study coordinators. Participants completed a standardized questionnaire that included medical history and coronary risk factors. Hypertension was defined by current use of antihypertensive drugs or physician-diagnosed hypertension. Diabe-
Table 1. Prevalence of obesity based on BMI versus WC

\begin{tabular}{|c|c|c|}
\hline \multirow[t]{2}{*}{ Definition } & \multicolumn{2}{|c|}{ Subjects, $n$ (\%) } \\
\hline & nonobese & obese \\
\hline $\begin{array}{l}\mathrm{BMI}, \geq 27.5 \mathrm{~kg} / \mathrm{m}^{2} \\
\text { WC }\end{array}$ & $846(87.2)$ & $124(12.8)$ \\
\hline $\begin{array}{l}>90 \mathrm{~cm} \text { in males } \\
>80 \mathrm{~cm} \text { in females }\end{array}$ & $623(64.3)$ & 347 (35.7) \\
\hline
\end{tabular}

tes mellitus was defined by the current use of antidiabetic agents or physician-diagnosed diabetes mellitus. Dyslipidemia was defined by the current use of lipid-lowering agents or physician-diagnosed dyslipidemia. Smoking history was defined as ever smokers (former or current smokers) or never smokers. BMI was calculated as weight in kilograms divided by the square of height in meters. Sinus rhythm status was ascertained by resting electrocardiogram. Clinical data were obtained on the same day as assessment of echocardiography and serum collection. WC was obtained $2.5 \mathrm{~cm}$ above the umbilicus, an anatomical landmark associated with abdominal fat mass measured by dual-energy $X$-ray absorptiometry [9].

We compared two definitions of obesity, namely: (1) BMI cutoff of $27.5 \mathrm{~kg} / \mathrm{m}^{2}$ as recommended by the World Health Organization for Asian populations [10] and (2) WC cut-offs of $>90 \mathrm{~cm}$ for males and $>80 \mathrm{~cm}$ for females, as recommended by the International Diabetes Federation Consensus Worldwide Definition of the Metabolic Syndrome [11]. Handgrip strength was measured from each participant using the Takei hand grip dynamometer (Model TKK5401 Grip D) and following standard protocols. Participants were instructed to stand upright with their arms let down naturally. The handgrip dynamometer was held with the indicator facing outwards, and the grip width was adjusted so that the second joint of the pointing finger made a right angle at the dynamometer. Participants were then instructed to clasp the grip with full force. Measurements obtained were recorded to the nearest $0.1 \mathrm{~kg}$. Two trials were performed for each hand, starting with the right hand. Only the highest value obtained from each hand was used. Overall handgrip strength was calculated as the mean of the maximum lefthand and right-hand grip strength measurements.

Echocardiography was performed using ALOKA $\alpha 10$ with a 3.5$\mathrm{MHz}$ probe. In each subject, standard echocardiography, which included 2-D, M-mode, pulse Doppler and tissue Doppler imaging, was performed in the standard parasternal and apical (apical 4-chamber, apical 2-chamber, and apical long) views, and three cardiac cycles were recorded. Left ventricular ejection fraction, left atrial (LA) volume, and LA volume index (LAVI) were measured. The trans-mitral flow $\mathrm{E}$ and $\mathrm{A}$ waves with the sample volume position at the tip of the mitral valve leaflets from the apical 4-chamber view were recorded by Doppler echocardiography. Myocardial relaxation (E/A) ratio was computed as a ratio of peak velocity flow in early diastole $\mathrm{E}(\mathrm{MV} \mathrm{E})(\mathrm{m} / \mathrm{s})$ to peak velocity flow in late diastole by atrial contraction A (MV A) (m/s). Pulsed wave tissue Doppler imaging was performed with the sample volume at the septal and lateral annulus from the apical 4-chamber view. The frame rate was between 80 and 100 frames per second. The tissue velocity patterns 
Table 2. Baseline characteristics based on different definitions of obesity

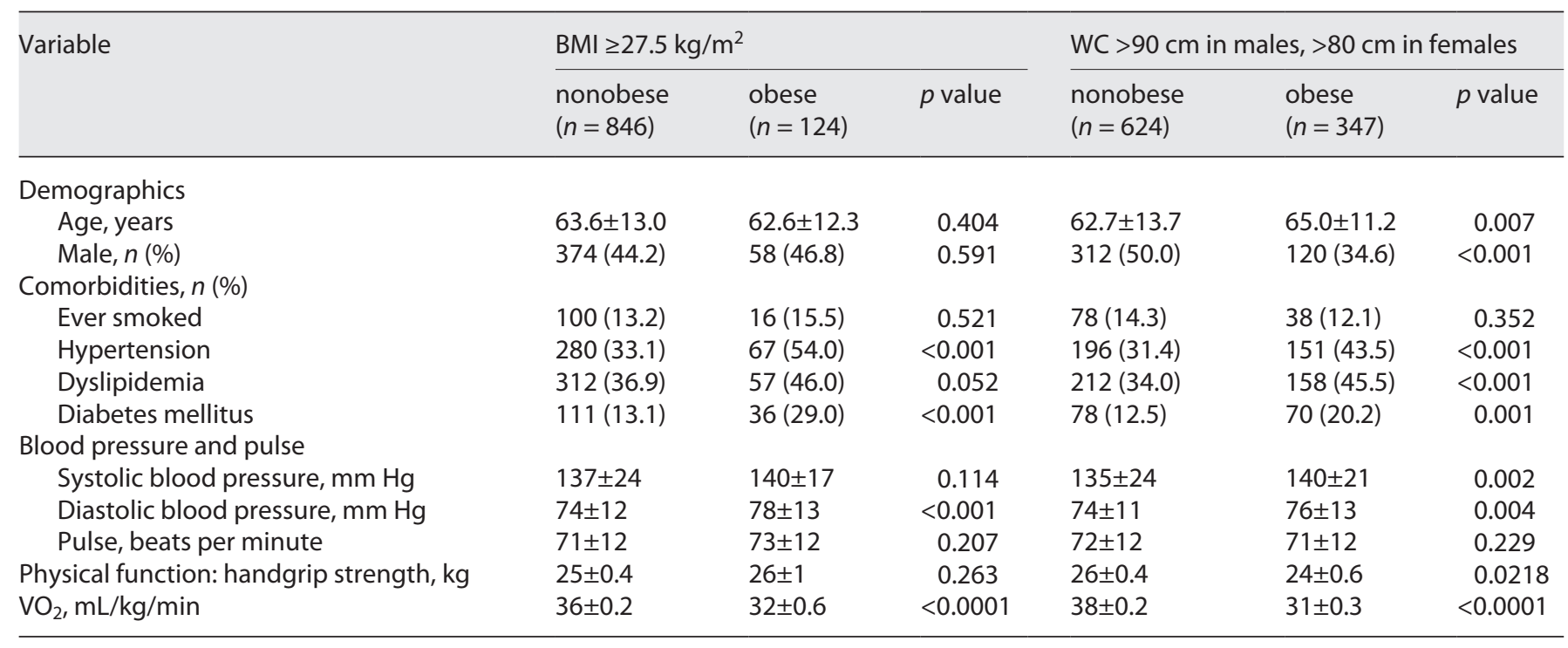

were recorded and expressed as E', and A'. All measurements were measured by the same operator and the measurements were averaged over three cardiac cycles and adjusted by the RR interval. The specific cardiovascular function of interest in this cohort of older adults was E/A properties, for which impairments in E/A, would suggest myocardial ageing [12]. E/A was defined by ratio of peak velocity flow in MV E to peak velocity flow in late diastole by $\mathrm{MV}$ $\mathrm{A}$, also referred to as the E/A ratio. MV E refers to the peak velocity of blood flow during early diastole from the left atrium into the left ventricle, where blood flows passively into the left ventricle during relaxation. MV A refers to the peak velocity of blood flow into the left ventricle in late diastole due to contraction of the left atrium. The echocardiography readers were blinded to the obesity status of the participants. We used a validated non-exercise prediction model comprising of physical activity questionnaire to estimate peak oxygen uptake $\left(\mathrm{VO}_{2}\right)$ milliliter/kilogram/minute $(\mathrm{mL} / \mathrm{kg} / \mathrm{min})[13,14]$, also previously used in this cohort [15].

\section{Statistics}

Clinical characteristics are presented as means and standard deviations for continuous data and frequency and percentage for categorical data. We determined agreement between BMI and WC definitions using Cohen's kappa. We compared demographics, clinical characteristics, and echocardiographic characteristics between nonobese and obese subjects based on either BMI or WC definitions. The Student's $t$ test was used for continuous data and the $\chi^{2}$ test was used for categorical data. Multiple linear regression analysis was subsequently performed to ascertain the relationship of cardiovascular structure and function to BMI and WC definitions, respectively. Variability of cardiovascular structure and function across BMI group and WC group were displayed in the error bar charts with standard error.
All statistical analyses were performed using STATA 15 (College Station, TX, USA). For all analyses, a two-tailed $p$ value of $<0.05$ was considered statistically significant.

\section{Results}

\section{Obesity Definitions}

Among 970 participants, 124 (12.8\%) were defined as obese by the BMI definition, while 347 (35.7\%) were defined as obese by the WC definition (Table 1). Inter-definitional agreement was fair between BMI and WC (Cohen's $\kappa=0.345)$.

Based on both definitions of BMI and WC, hypertension (54\% vs. $33 \%$; $p<0.001$ and $44 \%$ vs. $31 \%$; $p<0.001$ ) and diabetes mellitus (29\% vs. $13 \%$; $p<0.001$ and $20 \%$ vs. $13 \% ; p=0.001)$ were more prevalent among those defined as obese (Table 2). However, WC identified more women ( $65 \%$ vs. $50 \% ; p<0.001)$, older participants $(65 \pm 11$ vs. 63 \pm 14 years; $p=0.007)$ and dyslipidemic ( $46 \%$ vs. $34 \%$; $p<$ $0.001)$ participants as obese. Systolic blood pressure was also significantly higher (140 \pm 21 vs. $135 \pm 24 \mathrm{~mm} \mathrm{Hg} ; p$ $=0.002$ ) in obese versus nonobese participants defined by WC. Based on BMI, gender, age, dyslipidemia, and systolic blood pressure were not significantly different between obese and nonobese participants (Table 2). Participants defined as obese by WC definition had lower hand grip strength (24.2 vs. $25.9, p=0.022)$ compared to nonobese. On the other hand, participants defined as obese by 
Table 3. Key echocardiographic characteristics

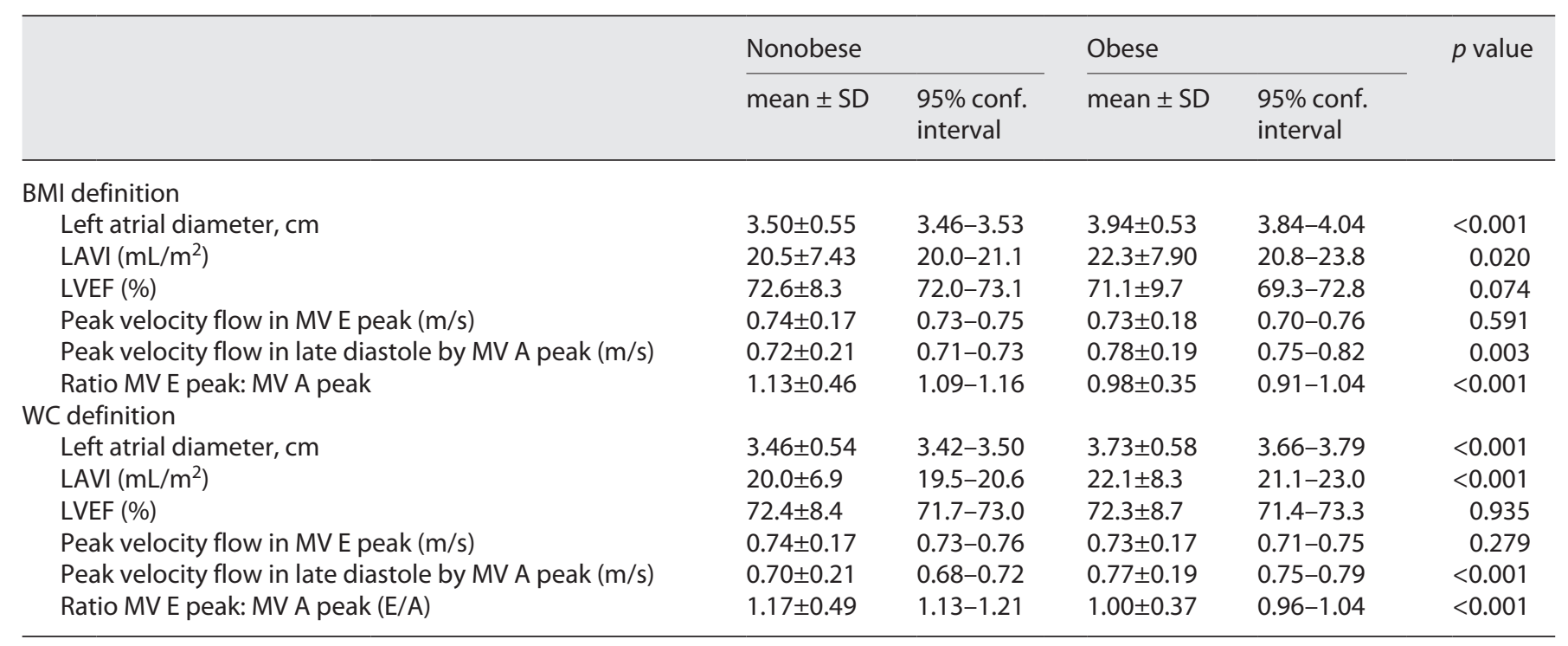

LVEF, left ventricular ejection fraction; SD, standard deviation.

Table 4. Multivariate regression model for E/A ratio

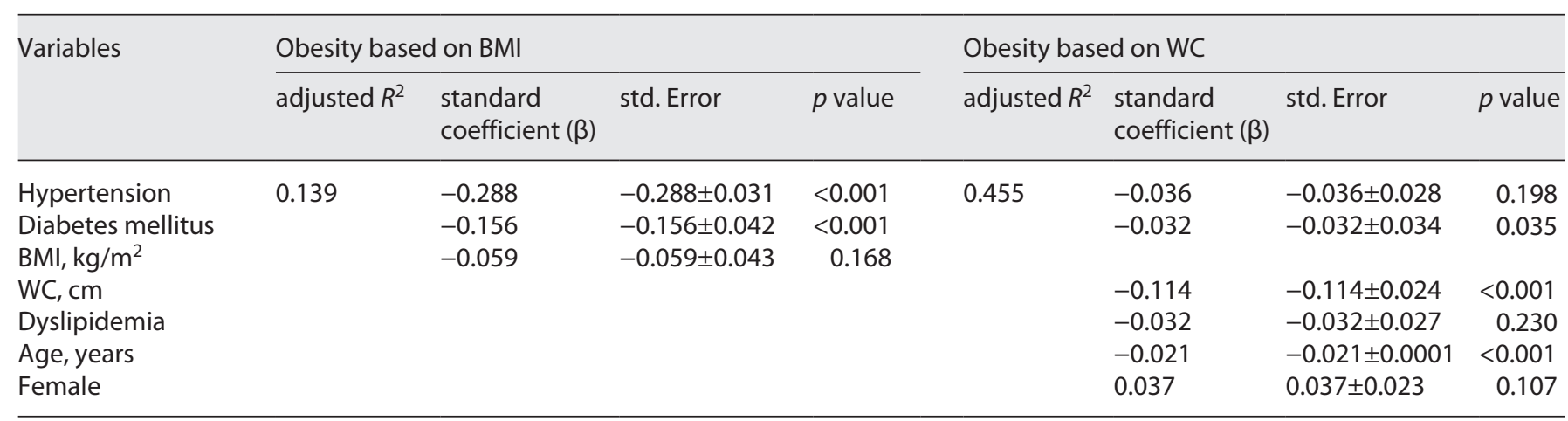

BMI definition had similar hand grip strength (26.4 vs. $25.2, p=0.26)$ compared to nonobese. Participants defined as obese by either WC or BMI definitions, had lower $\mathrm{VO}_{2}$, compared to nonobese participants (Table 2).

\section{Cardiovascular Structure and Function Based on \\ Obesity Definitions}

In general, participants who were defined by both BMI and WC as obese had larger left ventricular dimensions (online suppl. Table A; for all online suppl. material, see www.karger.com/doi/10.1159/000521729). Participants who were defined by BMI as obese had significantly lower E/A ratio compared to those who were not obese (1.13 \pm 0.46 vs. $0.98 \pm 0.35 ; p<0.001)$. Similarly, participants who were defined by WC as obese had significantly lower

Obesity in Older Adults with

Cardiovascular Ageing
E/A ratio compared to those who were not obese (1.17 \pm 0.49 vs. $1.00 \pm 0.37 ; p<0.001$ ) (Table 3). Left atrial size was also significantly larger in obese individuals in both the BMI group ( $3.94 \pm 0.53$ vs. $3.50 \pm 0.55 ; p<0.001)$ and WC group $(3.73 \pm 0.58$ vs. $3.46 \pm 0.54 ; p<0.001)$. The LAVI was also found to be significantly higher in obese individuals in both the BMI ( $22.3 \pm 7.90$ vs. $20.5 \pm 7.43$; $p$ $=0.020)$ and WC $(22.1 \pm 8.3$ vs. $20.0 \pm 6.9 ; p<0.001)$ groups. Left ventricular ejection fraction percentage was over $70 \%$ and not significantly different in both obese and nonobese for both BMI $(72.6 \pm 8.3$ vs. $71.1 \pm 9.7 ; p=$ $0.074)$ and WC (72.4 \pm 8.4 vs. $72.3 \pm 8.7 ; p=0.935)(\mathrm{Ta}-$ ble 3) (online suppl. Table A).

However, across high or low BMI categories, high WC was associated with more adverse mean $\mathrm{E} / \mathrm{A}$ and $\mathrm{VO}_{2}$ 


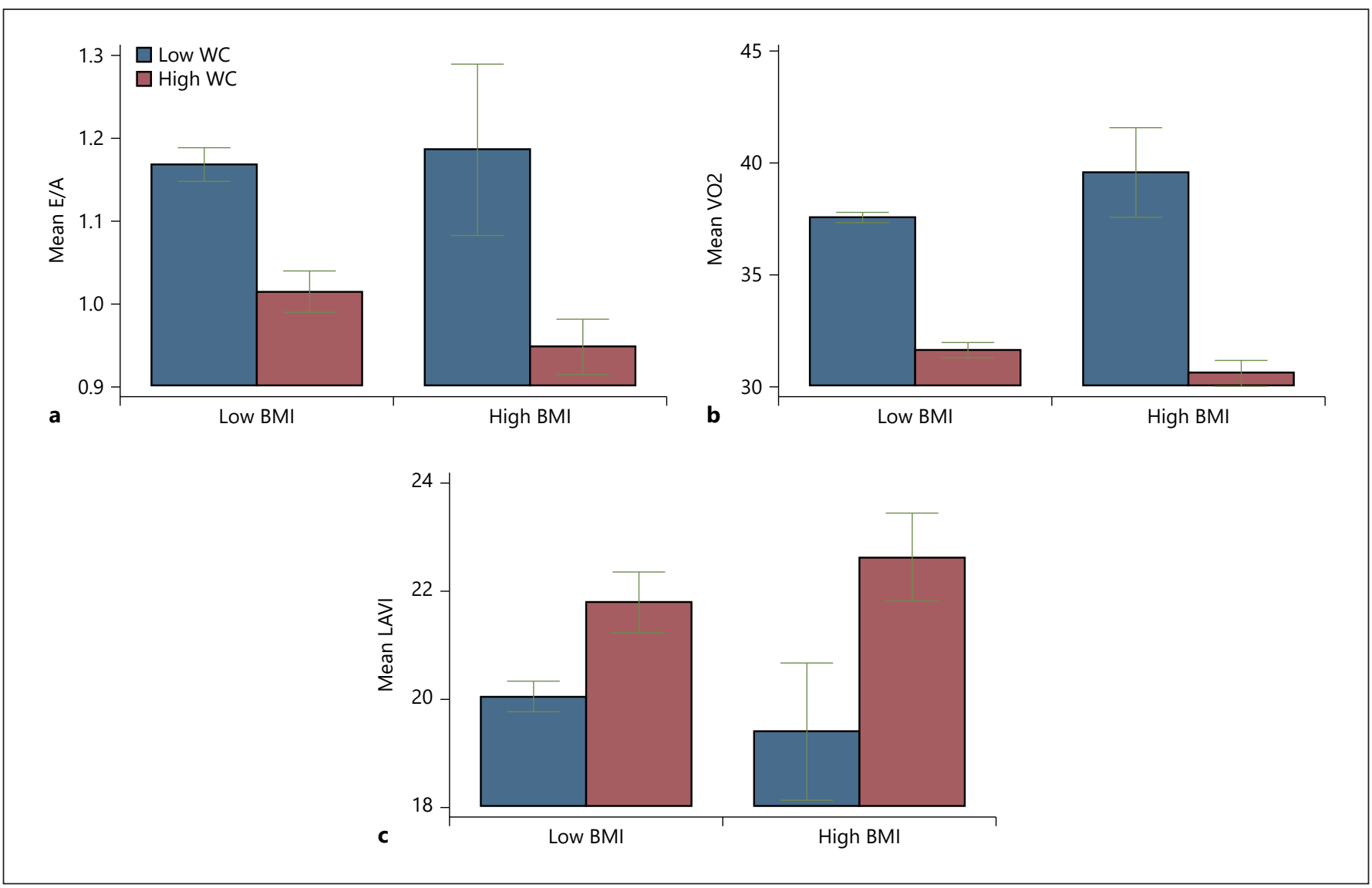

Fig. 1. Cardiovascular function and structure by BMI and WC. a E/A ratio (mean and standard error) by BMI and WC: across BMI categories, mean E/A was lower among those with high WC. *blue (mean E/A 1.17) versus red (mean E/A 1.01) (low BMI); $p<0.0001$; *blue (mean E/A 1.19) versus red (mean E/A 0.95) (high BMI); $p$ $=0.019$. $\mathbf{b} \mathrm{VO}_{2}$ (mean and standard error) by BMI and WC. Mean $\mathrm{VO}_{2}$ was lower among those with high $\mathrm{WC}$ *blue (mean $\mathrm{VO}_{2} 37.7$ )

measurements (Fig. 1a, b). Among those low BMI, high WC was associated with more adverse mean LAVI (Fig. 1c).

Multiple linear regression analysis was performed in $\mathrm{BMI}$ and $\mathrm{WC}$ groups to assess association of the $\mathrm{E} / \mathrm{A}$ ratio with obesity status after adjustment for significant covariates (Table 4). Adjusted $R^{2}$ value was $13.9 \%$ and $45.5 \%$ for BMI and WC groups, respectively. When adjusted for hypertension and diabetes mellitus, BMI was not associated with cardiovascular function. In contrast, WC was associated with $\mathrm{E} / \mathrm{A}(\beta=-0.114$, SE $-0.114 \pm 0.024, p<0.001)$, independent of age and diabetes mellitus. With each $1 \mathrm{~cm}$ increase in $\mathrm{WC}, \mathrm{E} / \mathrm{A}$ ratio declined by 0.114 . versus red (mean $\mathrm{VO}_{2}$ 31.7) (low $\left.\mathrm{BMI}\right) ; p<0.0001$; *blue (mean $\mathrm{VO}_{2}$ 39.7) versus red (mean $\mathrm{VO}_{2} 30.6$ ) (high BMI); $p<0.0001$. c LAVI (mean and standard error) by BMI and WC. Mean LAVI was larger among those with high WC despite low BMI. *blue (mean LAVI 20.0) versus red (mean LAVI 21.8) (low BMI); $p=0.003$; *blue (mean LAVI 19.4) versus red (mean LAVI 22.6) (high BMI); $p=0.18$.

\section{Discussion}

Based on a cohort of older adults, the prevalence of obesity varied depending on the definition used. The prevalence of obesity was higher at $35.7 \%$ based on WC, and only $12.8 \%$ based on BMI. Although both definitions identified more adverse alterations in cardiovascular structure and function, only WC was independently associated with impaired E/A. Importantly, even within nonobese BMI category, high WC was associated with impairments in E/A, aerobic capacity, and LA structure.

Comparing between definitions of BMI versus WC, WC identified the presence of obesity in adults who were older in age, whereas BMI did not differentiate between 
adults with older age. Although both definitions are intrinsically different and are not interchangeable, these prevalence rates highlight the importance of using appropriate definitions of obesity, particularly among older adults with aged biology.

The limited ability of BMI to identify obesity among older adults have been previously appreciated [16]. At extremes of age and weight, BMI has limited utility $[17,18]$. Apart from age, older adults have fluctuating body weights, related to ageing or accumulation of systemic illnesses $[19,20]$. We observed that those defined as obese by WC had lower hand grip strength, a possible reflection of concomitant muscle sarcopenia. Our findings concur with studies that have found associations between abdominal adiposity and poorer physical outcomes in sarcopenic adults [21]. This adds to the body of evidence that shows inverse associations between muscle strength and adiposity-related obesity markers, particularly among older adults [22-24].

Our findings are novel because they depict distinct associations between WC and cardiovascular structure and function among older adults. WC was linearly associated with impairments in myocardial function, namely E/A, a common early manifestation of myocardial ageing. Left atrial size was larger among the obese as defined by both $\mathrm{BMI}$ and WC definitions, a well-recognized risk factor for atrial fibrillation development $[25,26]$. While cardiometabolic complications of central adiposity are well established in current literature [27-29], our data provide a clue as to how obesity contributes to cardiovascular dysfunctions that may herald the onset and lead towards cardiovascular disease development.

The obesity-related risks of cardiovascular disease are well established $[2,30]$. While our cross-sectional study precludes causal inferences, the alterations in E/A and LA structure, point to specific key alterations in the cardiovascular system that are commonly involved in obesity-related heart failure and ageing, such as heart failure with preserved ejection fraction or atrial fibrillation among older adults. Importantly, we observed adverse alterations in E/A, LA structure, and aerobic capacity, among those with nonobese BMI but defined as obese by WC. Our observations are supported by recent studies that also reported metabolic abnormalities among individuals deemed to have normal BMI [18]. In a small study, women with normal BMI and high body fat percentage had lower resting metabolic rate and oxygen consumption, when compared to women with normal BMI and no excess in body fat percentage [31]. In a clinical study of heart failure patients, lean-fat patients with high waist to hip ratio and low BMI, had the worst outcomes at 1-year for heart failure hospitalization or mortality [32]. These observations should prompt intense efforts to address the early subclinical risks of atrial or ventricular dysfunction in older adults defined as obese by WC but lean by BMI.

From a clinical perspective, these results serve to emphasize the use of WC in addition to BMI, as a routine practice, particularly among older adults as well as among those defined as nonobese by BMI definition [23]. This may imply greater search or attention for cardiovascular dysfunction among those with high WC, in appropriate healthcare settings. In addition, therapeutic strategies against obesity may use these cardiovascular structural and functional features as targets useful for monitoring response to therapies, to reduce burdens of central obesity-related cardiovascular disease [33-35].

We acknowledge limitations in our study. In the absence of body fat measurements, the use of WC may only represent an incomplete measure of body fat composition. However, WC is not interchangeable with body fat [5]. Furthermore, for purposes of cardiovascular risk assessment, WC is an accepted marker of central adiposity [36]. Our findings are based on Asian older adults, utilizing obesity cut-offs based on prior Asian data. Hence our findings may not be extrapolated to cohorts of non-Asian descent. Importantly, we recognize that BMI may not be an accurate measure of obesity for Asians. The Asian phenotype of obesity comprises of higher proportions of visceral fat in the central abdominal regions ("central obesity") compared to Western populations [37-40]. Similar studies from other cohorts would be necessary to confirm our observations and improve generalizability. The observational study design does not imply causality between markers of obesity and cardiovascular function. Adaptive versus pathogenic responses cannot be differentiated based on this clinical study design. We did not correct for details such as medication data hence effects arising from medication treatment are unknown. This is a low-risk community cohort, hence marginal values in some of the observed measurements may reflect underestimation rather than overestimation of clinical significance. Even so, the large study sample provided reasonable sample power.

\section{Conclusion}

The prevalence of obesity varied depending on the definition used. WC identified higher prevalence of obesity, possibly related to central adiposity. Across BMI catego- 
ries, $\mathrm{WC}$ identified more adverse measurements in $\mathrm{E} / \mathrm{A}$, aerobic capacity, and LA structure. WC may better characterize the impact of obesity on cardiovascular ageing.

\section{Acknowledgments}

We thank the staff of the imaging laboratories for participating in the conduct of the study.

\section{Statement of Ethics}

Written informed consent was obtained from the participants upon enrolment. The SingHealth Centralised Institutional Review Board (CIRC/2014/628/C) had approved the study protocol.

\section{Conflict of Interest Statement}

The authors have no conflicts of interest to declare.

\section{Funding Source}

The Cardiac Aging Study has received funding support from the National Medical Research Council of Singapore $(\mathrm{MOH}-$ 000153), Hong Leong Foundation, Duke-NUS Medical School, Es- tate of Tan Sri Khoo Teck Puat, and Singhealth Foundation. Those participants recruited from the Singapore Chinese Health Study were supported by the United States National Institutes of Health (NIH R01 CA144034 and UM1 CA182876). The funders had no role in the design and conduct of the study; collection; management, analysis, and interpretation of the data; and preparation, review, or approval of the manuscript.

\section{Author Contributions}

A.S. Koh, J.P. Lim, and W.S. Lim conceptualized and designed the study. Y.H. Tan, B.M.H. Keng, F. Gao, L.L.Y. Teo, R.S. Tan, S.H. Ewe, and W.P. Koh performed data acquisition, analysis, and interpretation. Y.H. Tan, A.S. Koh, F. Gao, and W.S. Lim prepared the manuscript and figures.

\section{Data Availability Statement}

The data generated or analyzed are included in this article and or online supplementary files. Further inquiries can be directed to the corresponding author.

\section{Reference List}

1 Adams KF, Schatzkin A, Harris TB, Kipnis V, Mouw T, Ballard-Barbash R, et al. Overweight, obesity, and mortality in a large prospective cohort of persons 50 to 71 years old. N Engl J Med. 2006 Aug 24;355(8):763-78.

2 Kenchaiah S, Evans JC, Levy D, Wilson PW, Benjamin EJ, Larson MG, et al. Obesity and the risk of heart failure. N Engl J Med. 2002 Aug 1;347(5):305-13.

3 Stevens J, Cai J, Pamuk ER, Williamson DF, Thun MJ, Wood JL. The effect of age on the association between body-mass index and mortality. N Engl J Med. 1998 Jan 1;338(1): $1-7$.

4 Dhana K, Berghout MA, Peeters A, Ikram MA, Tiemeier H, Hofman A, et al. Obesity in older adults and life expectancy with and without cardiovascular disease. Int J Obes. 2016 Oct;40(10):1535-40.

5 Khor EQ, Lim JP, Tay L, Yeo A, Yew S, Ding YY, et al. Obesity definitions in sarcopenic obesity: differences in prevalence, agreement and association with muscle function. J Frailty Aging. 2020;9(1):37-43.

6 Gutierrez-Mariscal FM, Garcia-Rios A, Gomez-Luna P, Fernandez-Gandara C, Cardelo MP, Cruz-Ares S, et al. Age-dependent effect of metabolic phenotypes on carotid atherosclerotic disease in coronary heart disease pa- tients (CORDIOPREV Study). BMC Geriatr. 2020 Apr 22;20(1):151.

7 Koh AS, Gao F, Leng S, Kovalik JP, Zhao X, Tan RS, et al. Dissecting clinical and metabolomics associations of left atrial phasic function by cardiac magnetic resonance feature tracking. Sci Rep. 2018 May 25;8(1):813826456.

8 Hankin JH, Stram DO, Arakawa K, Park S, Low SH, Lee HP, et al. Singapore Chinese Health Study: development, validation, and calibration of the quantitative food frequency questionnaire. Nutr Cancer. 2001;39(2):18795.

9 Guerra RS, Amaral TF, Marques EA, Mota J, Restivo MT. Anatomical location for waist circumference measurement in older adults: a Preliminary Study. Nutr Hosp. 2012 Sep; 27(5):1554-61.

10 WHO Expert Consultation. Appropriate body-mass index for Asian populations and its implications for policy and intervention strategies. Lancet 2004 Jan 10;363(9403):15763.

11 Alberti KG, Zimmet P, Shaw J. Metabolic syndrome: a new world-wide definition. A consensus statement from the International Diabetes Federation. Diabet Med. 2006 May; 23(5):469-80.
12 Keng BMH, Gao F, Ewe SH, Tan RS, Teo LLY, Xie BQ, et al. Galectin-3 as a candidate upstream biomarker for quantifying risks of myocardial ageing. ESC Heart Fail. 2019 Oct; 6(5):1068-76.

13 Nes BM, Janszky I, Vatten LJ, Nilsen TI, Aspenes ST, Wisloff U. Estimating V.O 2peak from a nonexercise prediction model: the HUNT Study, Norway. Med Sci Sports Exerc. 2011 Nov;43(11):2024-30.

14 Nes BM, Vatten LJ, Nauman J, Janszky I, Wisløff U. A simple nonexercise model of cardiorespiratory fitness predicts long-term mortality. Med Sci Sports Exerc. 2014 Jun;46(6):1159-65.

15 Koh AS, Gao F, Tan RS, Zhong L, Leng S, Zhao X, et al. Metabolomic correlates of aerobic capacity among elderly adults. Clin Cardiol. 2018 Oct;41(10):1300-7.

16 Bales CW, Ritchie CS. Sarcopenia, weight loss, and nutritional frailty in the elderly. Annu Rev Nutr. 2002;22:309-23.

17 Nuttall FQ. Body mass index: obesity, BMI, and health: a critical review. Nutr Today. 2015 May;50(3):117-28.

18 Romero-Corral A, Somers VK, Sierra-Johnson J, Thomas RJ, Collazo-Clavell ML, Korinek J, et al. Accuracy of body mass index in diagnosing obesity in the adult general population. Int J Obes. 2008 Jun;32(6):959-66. 
19 Atti AR, Palmer K, Volpato S, Winblad B, De $\mathrm{RD}$, Fratiglioni L. Late-life body mass index and dementia incidence: nine-year follow-up data from the Kungsholmen Project. J Am Geriatr Soc. 2008 Jan;56(1):111-6.

20 Logroscino G, Sesso HD, Paffenbarger RS Jr, Lee IM. Body mass index and risk of Parkinson's disease: a Prospective Cohort Study. Am J Epidemiol. 2007 Nov 15;166(10):1186-90.

21 Lim JP, Chong MS, Tay L, Yang YX, Leung $\mathrm{BP}$, Yeo A, et al. Inter-muscular adipose tissue is associated with adipose tissue inflammation and poorer functional performance in central adiposity. Arch Gerontol Geriatr. 2019 Mar:81:1-7.

22 Jackson AW, Lee DC, Sui X, Morrow JR Jr, Church TS, Maslow AL, et al. Muscular strength is inversely related to prevalence and incidence of obesity in adult men. Obesity. 2010 Oct; 18(10):1988-95.

23 Keevil VL, Luben R, Dalzell N, Hayat S, Sayer AA, Wareham NJ, et al. Cross-sectional associations between different measures of obesity and muscle strength in men and women in a British Cohort Study. J Nutr Health Aging. 2015 Jan;19(1):3-11.

24 Volaklis KA, Halle M, Thorand B, Peters A, Ladwig KH, Schulz H, et al. Handgrip strength is inversely and independently associated with multimorbidity among older women: Results from the KORA-Age Study. Eur J Intern Med. 2016 Jun;31:35-40.

25 Casaclang-Verzosa G, Gersh BJ, Tsang TS. Structural and functional remodeling of the left atrium: clinical and therapeutic implications for atrial fibrillation. J Am Coll Cardiol. 2008 Jan 1;51(1):1-11.
26 Ayer JG, Almafragy HS, Patel AA, Hellyer RL, Celermajer DS. Body mass index is an independent determinant of left atrial size. Heart Lung Circ. 2008 Feb;17(1):19-24.

27 Harris TB, Ballard-Barbasch R, Madans J, Makuc DM, Feldman JJ. Overweight, weight loss, and risk of coronary heart disease in older women. The NHANES I Epidemiologic Follow-Up Study. Am J Epidemiol. 1993 Jun 15;137(12):1318-27.

28 Rexrode KM, Buring JE, Manson JE. Abdominal and total adiposity and risk of coronary heart disease in men. Int $J$ Obes Relat Metab Disord. 2001 Jul;25(7):1047-56.

29 Rimm EB, Stampfer MJ, Giovannucci E, Ascherio A, Spiegelman D, Colditz GA, et al. Body size and fat distribution as predictors of coronary heart disease among middle-aged and older US men. Am J Epidemiol. 1995 Jun 15;141(12):1117-27.

30 Bozkurt B, Aguilar D, Deswal A, Dunbar SB, Francis GS, Horwich T, et al. Contributory risk and management of comorbidities of hypertension, obesity, diabetes mellitus, hyperlipidemia, and metabolic syndrome in chronic heart failure: a scientific statement from the American Heart Association. Circulation. 2016 Dec 6;134(23):e535-78.

31 De LA, Martinoli R, Vaia F, Di RL. Normal weight obese (NWO) women: an evaluation of a candidate new syndrome. Nutr Metab Cardiovasc Dis. 2006 Dec;16(8):513-23.

32 Chandramouli C, Tay WT, Bamadhaj NS, Tromp J, Teng TK, Yap JJL, et al. Association of obesity with heart failure outcomes in 11 Asian regions: a Cohort Study. PLoS Med. 2019 Sep;16(9):e1002916.
33 Chrostowska M, Szyndler A, Hoffmann M, Narkiewicz K. Impact of obesity on cardiovascular health. Best Pract Res Clin Endocrinol Metab. 2013 Apr;27(2):147-56.

34 Dhaliwal SS, Welborn TA. Central obesity and multivariable cardiovascular risk as assessed by the Framingham prediction scores. Am J Cardiol. 2009 May 15;103(10):1403-7.

35 He X, Liu C, Chen Y, He J, Dong Y. Overweight without central obesity, cardiovascular risk, and all-cause mortality. Mayo Clin Proc. 2018 Jun;93(6):709-20.

36 Ross R, Neeland IJ, Yamashita S, Shai I, Seidell J, Magni P, et al. Waist circumference as a vital sign in clinical practice: a consensus statement from the IAS and ICCR working group on visceral obesity. Nat Rev Endocrinol. 2020 Mar;16(3):177-89.

37 Hu L, Huang X, You C, Li J, Hong K, Li P, et al. Prevalence of overweight, obesity, abdominal obesity and obesity-related risk factors in southern China. PLoS One. 2017;12(9): e0183934.

38 Misra A, Soares MJ, Mohan V, Anoop S, Abhishek V, Vaidya R, et al. Body fat, metabolic syndrome and hyperglycemia in South Asians. J Diabetes Complications. 2018 Nov; 32(11):1068-75.

39 Scott WR, Zhang W, Loh M, Tan ST, Lehne $B$, Afzal U, et al. Investigation of genetic variation underlying central obesity amongst South Asians. PLoS One. 2016;11(5): e0155478.

$40 \mathrm{Du}$ T, Sun X, Yin P, Huo R, Ni C, Yu X. Increasing trends in central obesity among Chinese adults with normal body mass index, 1993-2009. BMC Public Health. 2013 Apr 10; 13:327. 